\title{
Horizon universel, horizon régional Réseaux et territoires des commanderies hospitalières de Basse-Alsace au XIIIe et au XIVe siècle
}

\section{Nicolas Buchheit}

\section{OpenEdition}

\section{Journals}

Édition électronique

URL : http://journals.openedition.org/ifha/177

DOI : $10.4000 /$ ifha. 177

ISSN : 2198-8943

\section{Éditeur}

IFRA - Institut franco-allemand (sciences historiques et sociales)

\section{Édition imprimée}

Date de publication : 6 février 2011

Pagination : 133-141

ISSN : 2190-0078

\section{Référence électronique}

Nicolas Buchheit, « Horizon universel, horizon régional Réseaux et territoires des commanderies hospitalières de Basse-Alsace au XIIIe et au XIVe siècle », Revue de l'IFHA [En ligne], 3 | 2011, mis en ligne le 10 février 2012, consulté le 19 avril 2019. URL : http://journals.openedition.org/ifha/177 ; DOI : 10.4000/ifha. 177

Ce document a été généré automatiquement le 19 avril 2019.

(C)IFHA 


\title{
Horizon universel, horizon régional Réseaux et territoires des commanderies hospitalières de Basse-Alsace au XIIIe et au XIVe siècle
}

\author{
Nicolas Buchheit
}

\section{NOTE DE L'ÉDITEUR}

Nicolas Buchheit, professeur agrégé d'histoire au lycée March-Bloch de Bischheim, a soutenu sa thèse de doctorat sous la direction de Georges Bischoff le 17 novembre 2010, à l'université de Strasbourg.

$\mathrm{Au}$ cours du XIIIe et du XIVe siècle, la Basse-Alsace a compté quatre commanderies hospitalières : Dorlisheim, Rhinau, Sélestat et Strasbourg. L'ordre de l'Hôpital-de-SaintJean-de-Jérusalem s'est établi dans la région au début du XIIIe siècle, avec la fondation de la commanderie de Dorlisheim. Mais il faut attendre les années 1260 pour le voir continuer à se développer, avec les fondations des commanderies de Rhinau et de Sélestat. Par la suite, la suppression de l'ordre du Temple et la décision prise en 1312 par le pape Clément $\mathrm{V}$ de remettre ses biens aux Hospitaliers eurent des effets limités sur les commanderies de Basse-Alsace : le nombre de commanderies templières dans la région était réduit (Baumgarten, Bergheim) et seul le temporel de la commanderie templière de Bergheim fut attribué à la commanderie hospitalière de Sélestat. Cependant, de profondes transformations survinrent à partir du milieu du XIVe siècle. La commanderie de Rhinau fut tout d'abord rattachée à celle de Dorlisheim dont elle devint un membre. Dorlisheim fit aussi l'acquisition en 1354 de la paroisse Saint-Georges de Haguenau, où une maison de frères se constitua. Puis la fondation de la commanderie « Zum Grünen Wörth » - « En l'îleverte» ou «Au Marais-vert»- de Strasbourg, en 1370-1371, apporta de nouveaux 
changements dans ce réseau d'établissements où elle devait s'insérer. La commanderie de Dorlisheim lui donna la maison de Rhinau en 1374, avant que celle-ci ne fût détruite par une inondation du Rhin au tournant du siècle suivant. Enfin, la commanderie de Sélestat et son membre Bergheim lui furent attribués en 1399, au terme d'une réorganisation des établissements de l'ordre en Basse-Alsace, progressivement polarisé sur la commanderie de Strasbourg.

Les commanderies constituaient l'échelon institutionnel et territorial de base de l'ordre de l'Hôpital. Elles sont apparues en Occident très peu de temps après sa création au début du XIIe siècle à Jérusalem, dans le contexte de la première croisade. Leur fonction était d'administrer les biens que l'ordre avait acquis par des donations ou des achats, et en tirer des revenus. Elles servaient à fournir des subsides annuels (responsiones) et à recruter des hommes pour les besoins de l'ordre dans sa mission d'accueil et de soin des pèlerins en Terre sainte, mission à laquelle s'est ajoutée au cours du XIIe siècle la défense des Lieux saints et des États latins d'Orient. Mais, depuis la défaite d'Hattin en 1187 et la prise de Jérusalem par Saladin la même année, la quasi-disparition du royaume latin de Jérusalem entraîna le déplacement du siège de l'Hôpital dans la ville d'Acre. Lorsque les commanderies hospitalières apparurent en Basse-Alsace, l'ordre poursuivait la défense de la principauté d'Antioche, du royaume arménien de Cilicie, du comté de Tripoli, du royaume de Jérusalem, partiellement reconstitué dans la première moitié du XIIIe siècle et, dans une moindre mesure, des royaumes ibériques. Des études récentes ont montré sa capacité à reconstituer ses fonds et ses contingents tout au long du XIIIe siècle, grâce au patrimoine des commanderies en Occident ${ }^{1}$. La fin des États latins d'Orient, après la prise d'Acre en 1291 par les Mamelouks, contraignit les Hospitaliers à se replier sur le royaume de Chypre. Malgré les critiques dont les ordres religieux-militaires furent l'objet dans la seconde moitié du XIIIe siècle, ils gardaient une place importante dans les projets de récupération de la Terre sainte. Au cours de cette époque troublée, dont les Templiers furent les victimes à partir de leur arrestation en 1307, les Hospitaliers firent la conquête de Rhodes de 1306 à 1310, d'où ils poursuivirent leurs missions jusqu'à la prise de l'île par le sultan Soliman en 1522, avant qu'ils ne s'établissent sur l'île de Malte jusqu'en 1798. À Rhodes, malgré leurs difficultés financières récurrentes et la dépression économique qui affectait les commanderies en Occident, les Hospitaliers constituèrent une principauté ecclésiastique dont ils devaient assurer la défense et depuis laquelle ils affrontèrent les Turcs et les Mamelouks, sous l'incitation des papes qui faisaient de l'ordre un élément encore déterminant de leurs projets de croisade.

Le développement du réseau des commanderies hospitalières en Basse-Alsace s'inscrit donc dans ce contexte lointain auquel il était lié, fait de difficultés et de crises malgré lesquelles l'ordre parvint à préserver et à renouveler sa raison d'être. Ce développement est cependant relativement tardif, en comparaison avec celui qu'il a connu dans le sud et l'ouest de l'Europe et qui s'achève le plus souvent à l'aube du XIIIe siècle. À cet égard, la fondation de la commanderie de Strasbourg dans la seconde moitié du XIVe siècle paraît d'autant plus anachronique. Outre cette singularité chronologique, ce réseau eut, au cours des XIIIe et XIVe siècles, une emprise territoriale qui le distinguait des régions limitrophes de la vallée du Rhin supérieur, où l'ordre était aussi installé. L'implantation des commanderies de Basse-Alsace contrastait tout particulièrement avec le semis resserré des établissements qui s'était constitué dans l'oberrhein, au sud de la limite ancienne du Landgraben qui séparait la Basse de la Haute-Alsace. En aval, la documentation montre que l'expansion des commanderies de Basse-Alsace ne dépassait 
pas la forêt de Haguenau. À l'ouest, les possessions de ces maisons sont restées en deçà de la frontière linguistique entre le français et l'allemand, laquelle traversait la Lorraine. Enfin, à l'est, sur la rive droite du Rhin, l'Ortenau était un autre terrain d'expansion de ces commanderies, au-delà duquel elles n'ont pas progressé.

Cette inscription régionale des commanderies hospitalières de Basse-Alsace aux XIIIe et XIVe siècles fut le résultat des interactions de plusieurs réseaux. Tout d'abord, celui formé par les commanderies entre elles, si elles ne devaient être comprises que comme de simples maisons conventuelles, mais aussi avec tout ce qui dépendait d'elles, dès lors qu'elles sont envisagées comme "un mécanisme institutionnel $»^{2}$. Ensuite, le réseau qui les plaçait au niveau inférieur d'un ordre dont les échelons hiérarchiques supérieurs étaient constitués du prieuré d'Allemagne - représentant l'autorité du couvent central et du grand-maître de l'ordre - celui-ci ne relevant que du souverain pontife. Enfin, le réseau qui les mettait en relation avec la société locale environnante, dont leur dynamisme et leurs activités procédaient. De ce fait, les commanderies furent des interfaces entre ces différents acteurs, des lieux où intervenaient, par définition, des phénomènes d'exploitation et de transformation des uns par les autres : elles formaient donc des territoires réticulaires sous-tendus par un espace régional. Ainsi, leur géographie incite à s'interroger sur la participation d'un ordre religieux-militaire, dont l'horizon d'action était « universel », à une construction régionale dans la vallée du Rhin supérieur.

Comme il ne s'agissait pas d'appréhender l'ordre de l'Hôpital dans ses multiples défis pour préserver une unité entre ses différentes composantes "nationales» ou « régionales », le terme "universel » a été préféré à ceux de "supranational» ou de "suprarégional » pour le qualifier. En effet, par « universel », on entend, à la suite de Carlos de Ayala Martínez $z^{3}$ que les Hospitaliers dépendaient de l'universalisme pontifical, mis en action, au travers de la croisade, dans la défense et l'expansion de la chrétienté : un horizon idéologique, mais dont la réalité physique pour les commanderies de BasseAlsace se manifestait, à l'échelle régionale, au moins par l'envoi de ressources matérielles et humaines vers l'Orient lointain. Les commanderies étaient le lieu d'articulation entre ces deux dimensions, évoquées dans un article fondateur de Kaspar Elm, qui présentait en 1994 les ordres religieux-militaires comme une forme de vie religieuse «entre universalisme et particularisme $»^{4}$. Celui-ci amena rapidement le débat historiographique à prendre en considération la relation de ces ordres religieux avec le fait régional, le problème étant étendu aux ordres mendiants, dont les modes de fonctionnement sont régulièrement apparentés ${ }^{5}$. Néanmoins, si l'emprise régionale est souvent bien explorée, les tensions qu'elle pouvait provoquer avec un horizon universaliste le sont bien moins et n'ont été le sujet que d'un nombre limité de travaux, portant sur les ordres mendiants ${ }^{6}$.

Rendre compte des réseaux et des territoires des commanderies nécessite de se pencher sur toutes les sources disponibles les concernant, à savoir près de 750 actes de la pratique, 85 sceaux, des documents de gestion, des documents nécrologiques - deux des quatre obituaires qui subsistent des ordres religieux-militaires concernent les commanderies hospitalières de Basse-Alsace - et des textes narratifs. Presque la totalité de cette documentation est conservée dans des fonds d'archives alsaciens, principalement ceux des archives départementales du Bas-Rhin et des archives municipales de Haguenau, dans une moindre mesure des archives de la ville et de la communauté urbaine de Strasbourg, ainsi que de la Bibliothèque nationale et universitaire de Strasbourg. L'étude s'appuie aussi sur des vestiges monumentaux. Plusieurs instruments de travail ont été constitués : 
un catalogue prosopographique des frères, des donnés et du personnel des commanderies, suivi de tableaux synoptiques et de cartes; un catalogue des sceaux; un inventaire des actes des papes, des grands-maîtres et des prieurs concernant les commanderies, amenant à constituer un tableau chronologique de leurs interventions ; des graphiques et des cartes de localisation sur le temporel des commanderies, ainsi que les donateurs et les vendeurs.

Par-delà une monographie supplémentaire, la question de la présence des commanderies hospitalières en Basse-Alsace, envisagée sous l'angle des réseaux et des territoires, espère apporter quelques perspectives sur l'intégration régionale d'un ordre universel. Depuis l'apparition de l'ordre dans la région au début du XIIIe siècle jusqu'à sa polarisation strasbourgeoise à la fin du XIVe siècle, trois moments se sont succédés dans l'histoire des commanderies tout en se chevauchant. À ces trois périodes correspond respectivement un marquage régional, selon trois échelles géographiques différentes et une progressive focalisation. Ainsi, le temps des fondations et du premier développement au XIIIe siècle et au début du XIVe siècle est nourri par les évolutions qui affectaient les pays rhénans, où les princes territoriaux rencontraient l'affirmation croissante des villes et de la petite noblesse. Croissance, crises et adaptations, depuis la fin du XIIIe siècle jusque dans la seconde moitié du XIVe siècle, caractérisent ensuite des commanderies qui s'enracinaient plus profondément dans la Basse-Alsace. Enfin, la seconde moitié du XIVe siècle se différenciait par la volonté de réforme qui animait alors le prieuré d'Allemagne, mais gardait nombre d'aspects de la période précédente en héritage, à la faveur d'une société strasbourgeoise hégémonique.

L'apparition et le premier développement des commanderies hospitalières en BasseAlsace, depuis le début du XIIIe siècle jusqu'au début du XIVe siècle, furent d'abord le résultat de politiques territoriales. Ces établissements religieux servirent à la fois à maîtriser des sites de communication et des villes en pleine croissance, nouveaux lieux de pouvoir. Par les fonctions centrales qu'elles exerçaient, les commanderies contribuaient alors à l'organisation d'une région aux limites encore floues, mais dont la principale frontière était constituée par la limite linguistique entre parlers romans et germaniques. Cette géographie était l'expression des réseaux qui avaient favorisé les commanderies. Après avoir été soutenues par des pouvoirs princiers, notamment les évêques de Strasbourg, jusqu'au début des années 1270, elles furent l'expression d'un besoin social dans une petite noblesse - ainsi qu'une bourgeoisie qui cherchait à s'en rapprocher - en pleine aristocratisation, que la rencontre avec les commanderies et les valeurs chevaleresques qu'elles incarnaient permettait d'affirmer et de maintenir.

La prise en main de ces établissements par la petite noblesse provoqua un resserrement de leurs territoires, à partir du second quart du XIVe siècle. D'un espace rhénan, les commanderies passaient à un développement contenu dans la Basse-Alsace, tant par l'origine des frères qui y vivaient que par leur rôle d'encadrement social auprès des donnés - des semi-religieux qui s'affiliaient à l'ordre -, des fidèles et des dépendants, ou que par la gestion de leur temporel inscrite dans les réalités économiques locales. Cet enracinement régional fut renforcé par les groupes parentaux qui firent des commanderies des moyens d'expression de leurs solidarités et de leur pouvoir. Au début $\mathrm{du}$ XIVe siècle, la commanderie de Dorlisheim avait encore une position centrale dans le prieuré d'Allemagne et la commanderie de Sélestat resta par la suite davantage contrôlée par les hauts dignitaires du prieuré. Mais les difficultés et les échecs que les Hospitaliers subirent pour faire valoir leurs droits sur les biens du Temple, supprimé en 1312, 
montrent que l'autorité des échelons hiérarchiques supérieurs de l'ordre était mise à l'épreuve : seule la commanderie templière de Bergheim fut acquise par les Hospitaliers et remise à la commanderie de Sélestat. Quant à la commanderie de Baumgarten, située au sud-ouest de Brumath, ses biens furent totalement usurpés par des seigneurs locaux. Enfin, l'attribution de la paroisse Saint-Georges de Haguenau à la commanderie de Dorlisheim, par décision du roi Charles IV en 1354, mettait un terme à une longue attente de l'ordre, auquel le patronage avait été donné en 1262. L'entremise possible de l'évêque de Strasbourg Johannes de Lichtenberg, dernier représentant d'une famille de la région à la tête du diocèse, montre que les réseaux régionaux de l'ordre ont pu agir en sa faveur.

Depuis le début du XIVe siècle, les commanderies étaient touchées par une autre dynamique : leur progressive intégration à la ville de Strasbourg, selon trois modalités qui se sont succédées, par un rapport de cause à effet, et ajoutées. Tout d'abord, une attraction et un marquage de la ville sur les commanderies. Des familles strasbourgeoises avaient besoin de ces établissements religieux dans leur affirmation aristocratique et avaient les moyens financiers pour les maîtriser. Puis vint la fondation d'une commanderie dans la ville en 1370-1371, qui devint un élément de l'identité urbaine de Strasbourg. Elle avait été le résultat conjoint du projet du bourgeois Rulman Merswin de se retirer dans un établissement religieux pour y vivre son idéal mystique et celui du prieur d'Allemagne, Konrad de Braunsberg, qui cherchait à réformer matériellement et moralement son prieuré. Enfin, l'acquisition d'un temporel et la prise en main de domaines de l'ordre par la commanderie strasbourgeoise à la fin du XIVe siècle est révélatrice d'une hégémonie de la métropole, du moins d'une prétention de Strasbourg à la domination sur un territoire qui avait des similarités avec celui du diocèse de Strasbourg. En certains lieux, les limites diocésaines n'étaient pas toujours infranchissables pour l'établissement, en d'autres, la commanderie ne put se développer. Nonobstant, à la fin du XIVe siècle, la territorialité de la commanderie de Strasbourg participait pleinement à la définition de la métropole strasbourgeoise dans le fossé rhénan.

En dernière analyse, le premier apport de ce travail est un établissement plus précis, sans être définitif, de l'histoire des commanderies hospitalières de la région au XIIIe et au XIVe siècle. C'est l'interprétation de ces établissements sous l'angle des réseaux et les territoires qui a permis d'obtenir ce résultat. Plus encore, cette démarche a mis en évidence des ressorts et des effets de développement pour une grande part inédits.

- Les dynamiques de ces maisons religieuses relevaient tout d'abord de multiples stratégies de domination à l'échelle locale, d'abord de la haute aristocratie, puis de la petite noblesse et de la bourgeoisie. C'est aussi ce qui est perceptible dans l'inscription territoriale des commanderies, sur des sites de communication et dans des espaces urbains en formation, ainsi que par leur rôle structurant dans la région.

- Le facteur régional a eu une action déterminante. On passe d'un développement dont les caractéristiques sont propres au monde rhénan à une évolution plus circonscrite à la Basse-Alsace, à partir de la fin du XIIIe siècle, et pour finir à une progressive emprise strasbourgeoise. Cela se fit au détriment d'un horizon universel qui s'éloigne de plus en plus des réalités quotidiennes de ces établissements. Il ne faut cependant pas voir dans ce phénomène les prémices d'une construction identitaire régionale, dont la conscience et l'expression sont plus tardives. Les commanderies hospitalières de Basse-Alsace avaient certes des territorialités qui respectaient les limites traditionnelles d'une région, mais elles étaient aussi très ouvertes. En revanche, ces établissements ont participé au moins à 
deux constructions régionales : tout d'abord, l'affirmation croissante dans la vie sociale de la frontière linguistique entre les parlers romans et les parlers germaniques, frontière devenue structurelle pour les commanderies et qui amène à s'interroger sur le rôle et la pratique des langues dans l'organisation générale de l'ordre de l'Hôpital; la seconde construction est celle de la métropole strasbourgeoise, dont l'action permit de singulariser une région dans le fossé rhénan.

- Des communautés parentales ou urbaines ont eu progressivement davantage d'emprise sur ces établissements. À un ordre qui se pense comme une communauté de frères au XIIIe siècle succède un ordre patrimonialisé, voire communalisé au XIVe siècle, notamment dans le cas de la commanderie de Strasbourg. Il est difficile de comprendre quelles furent les causes de ce passage d'un état à un autre et son acceptation par les autorités de l'ordre. Il pourrait être lié au fait que ces établissements ont été pris en main par une petite noblesse et une bourgeoisie qui en avaient besoin pour affirmer leur position dans la société environnante. Il conviendrait de mettre cela en perspective avec la cléricalisation de l'ordre au XIVe siècle, avec la présence très forte de semi-religieux, dont des femmes, dans les commanderies. Enfin, rentes et prébendes ont poussé à fixer des frères dans une région d'où ils étaient originaires et à entretenir des relations avec leurs parents.

Au final, les commanderies hospitalières apparaissent comme des espaces de domination sociale : ce travail est en définitive une géographie de la domination au Moyen Âge. Cela ne remet pas en question la fonction logistique des commanderies. Celle-ci est en effet partie prenante d'un système où se confondent l'universalisme chrétien et des stratégies de prééminence sociale et territoriale. Entre ces deux pôles, il y eut des tensions et des déséquilibres. En ce sens, la fondation de la commanderie strasbourgeoise constitue une particularité : elle fut la manifestation réunie de l'universel et du régional, l'une et l'autre à des fins de domination.

\section{NOTES}

1. Cf. Judith Bronstein, The Hospitallers and the Holy Land. Financing the Latin East, 1187-1274, Woodbridge : Boydell Press, 2005.

2. Philippe Josserand, article "Commanderie» in: Nicole Bériou, Philippe Josserand (dir.), Prier et combattre. Dictionnaire européen des ordres militaires au Moyen Âge, Paris : Fayard, 2009, p. 245.

3. Carlos de Ayala Martínez, Las órdenes militares hispánicas en la Edad Media (siglos XIIXV), Madrid: M. Pons Historia, 2003, p. 21-22 ; Id., «Les ordres militaires dans l'Europe médiévale: origine, signification, typologie " in: Carlos de Ayala Martínez, Feliciano Novoa Portela (dir.), Le Glaive et la croix. Templiers, hospitaliers, chevaliers teutoniques et autres ordres militaires au Moyen Âge, Paris : Mengès, 2005, p. 15. 
4. Kaspar Elm, «Gli ordini militari. Un ceto di vita religiosa fra universalismo e particularismo » in : Enzo Coli, Maria De Marco, Francesco Tommasi (dir.), Militia sacra. Gli ordini militari tra Europa e Terrasanta, Perugia : Libreria L'Altra, 1994, p. 9-28.

5. Zenon Hubert Nowak (dir.), Ritterorden und Region..., Toruf: Uniw. Mikołaja Kopernika (Ordines militares. Colloquia Torunensia Historica, 8), 1995 ; Jürgen Sarnowsky (dir.), Mendicants, Military Orders, and Regionalism in Medieval Europe, Aldershot: Ashgate, 1999.

6. Cf. Anne Müller, « Nationale Abgrenzung in universalen Verbänden? Zur Entwicklung und Autonomiebestrebung der schottischen Franziskaner im 13. und 14. Jahrhundert " in : Reinhardt Butz, Jörg Oberste (dir.), Studia monastica. Beiträge zum klösterlichen Leben im Mittelalter, Münster : LIT (Vita regularis. Ordnungen und Deutungen religiösen Lebens im Mittelalter, 22), 2004, p. 261-285. L'auteur appuie notamment sa réflexion sur J. Sarnowsky, Mendicants ..., op. cit., et Annette Kehnel, Regionale Ordnungen universaler Konzepte. Die Franziskaner auf den Britischen Inseln (13.-16. Jh.). Historische Fallstudie zu einer europäischen Gemeinschaft des Mittelalters, HDR, TU Dresden, 2003. 